\title{
Cenários de Aprendizagem na Formação Inicial de Professores de Informática
}

\author{
Ana Pedro, João Piedade \& João Filipe Matos
}

\begin{abstract}
Resumo:
Esta investigação visa analisar as relações entre o modelo pedagógico seguido na formação inicial de professores, no que se refere à indução na prática pedagógica, e as opções pedagógicas realizadas pelos futuros professores na prática pedagógica supervisionada. Assume-se que através da análise às escolhas efetuadas pelos futuros professores de informática, no domínio das metodologias de ensino utilizadas, é possível caracterizar o modelo pedagógico de iniciação à prática profissional baseado no desenho de cenários de aprendizagem na formação inicial de professores utilizado no curso de Mestrado de Ensino de Informática da Universidade de Lisboa. Para tal, foram analisados 56 relatórios de prática de ensino supervisionada desenvolvidos desde 2011, através de uma metodologia bibliométrica de recolha de dados, a fim de caracterizar as opções pedagógicas e didáticas tomadas pelos futuros professores relativamente ao planeamento e implementação da prática pedagógica supervisionada. Os resultados identificaram a relação entre o modelo pedagógico seguido na formação inicial de professores e as opções pedagógicas tomadas pelos futuros professores na prática pedagógica supervisionada.
\end{abstract}

Palavras-chave:

formação inicial de professores; metodologias de ensino e de aprendizagem; cenários de aprendizagem ensino de informática. 


\title{
Learning Scenarios in Computer Science Initial Teacher Education
}

\begin{abstract}
This research aims to establish a relationship between the pedagogical model followed in the initial teacher education, regarding the induction in pedagogical practice, and the pedagogical options made by the future teachers in supervised pedagogical practice. It is assumed that through the analysis of the choices made by future teachers in the field of teaching methodologies used, it is possible to characterize the pedagogical model of professional practice initiation, based on the design of learning scenarios in teacher education used in the Master Degree in Computer Science Teaching at the Universidade de Lisboa. For this purpose, we analyzed 56 reports, developed since 2011, using a bibliometric methodology of data collection, aiming to characterize the pedagogical and didactic options taken by the future teachers regarding the planning and implementation of supervised pedagogical practice. The results identified a relationship between the pedagogical model followed in initial teacher education and the pedagogical options taken by the future teachers in their supervised pedagogical practice.
\end{abstract}

Keywords: initial teacher education; teaching and learning methodologies; learning scenarios; computer science education.

\section{Scénarios d'apprentissage dans la formation initiale des enseignants en informatique}

Résumé : Cette recherche a l'intention d'établir une relation entre le modèle pédagogique suivi dans la formation initiale des enseignants, en ce qui concerne l'incorporation dans la pratique pédagogique, et les options pédagogiques proposées par les futurs enseignants dans la pratique pédagogique supervisée. On suppose qu'en analysant les choix des futurs enseignants en informatique dans le domaine des méthodologies d'enseignement utilisées, il est possible de caractériser le modèle pédagogique d'initiation à la pratique professionnelle basé sur la conception de scénarios d'apprentissage dans la formation initiale des enseignants utilisée dans le Maîtrise en Enseignement de I' Informatique à l'Universidade de Lisboa. À cette fin, 56 rapports de pratiques supervisées élaborés depuis 2011 ont été analysés à lıaide d’une méthodologie bibliométrique de collecte de données, visant à caractériser les options pédagogiques et didactiques des futurs enseignants en ce qui concerne la planification et la mise en œuvre de pratiques pédagogiques supervisées. Les résultats trouvés ont identifié une relation entre le modèle pédagogique suivi dans la formation initiale des enseignants et les options pédagogiques par les futurs enseignants dans la pratique pédagogique supervisée.

Mots-clés: formation initiale d'enseignants; rapports de pratique supervisée; éducation informatique; méthodologies d'enseignement et d'apprentissage; scénarios d'apprentissage.

\section{Escenarios de aprendizaje en la formación inicial de profesores de informática}

Resumen: En esta investigación procura establecerse una relación entre el modelo pedagógico seguido en la formación inicial de profesores, en lo que se refiere a la inducción en la práctica pedagógica, y las opciones pedagógicas realizadas por los futuros profesores en la práctica pedagógica supervisada. Se asume que, a través del análisis a las elecciones efectuadas por los futuros profesores de informática, en el dominio de las metodologías de enseñanza utilizadas, es perecedero caracterizar el modelo pedagógico de iniciación a la práctica profesional basado en el diseño de escenarios de aprendizaje en la formación inicial de profesores utilizado en el curso Informática Maestro de Educación de la Universidad de Lisboa. Para ello, se analizaron 56 informes de práctica supervisada desarrollados desde 2011, a través de una metodología bibliométrica de recogida de datos, buscando caracterizar las opciones pedagógicas y didácticas tomadas por los futuros profesores en cuanto a la planificación e implementación de la práctica pedagógica supervisada. Los resultados encontrados identificaron la relación entre el modelo pedagógico seguido en la formación inicial de profesores y las opciones pedagógicas tomadas por los futuros profesores en la práctica pedagógica supervisada.

Palabras clave: formación inicial de profesores; informes de práctica supervisada; metodologías de enseñanza y aprendizaje; escenarios de aprendizaje; enseñanza de informática. 
Os modelos de formação inicial de professores (FIP) têm vindo a sofrer alterações, de diferentes âmbitos, ao longo das últimas décadas. Não é nossa intenção analisar e discutir estas alterações, mas sim explicitar, o atual modelo de formação de professores em Portugal, decorrente da reformulação proposta pelo processo de Bolonha, tomando como exemplo o modelo organizacional da FIP de Informática, para o $3^{\circ}$ ciclo do ensino básico e ensino secundário, implementado na Universidade de Lisboa. Pretende-se assim estabelecer uma relação entre o modelo pedagógico seguido na FIP nesse curso, no que se refere à indução na prática pedagógica, e as opções pedagógicas realizadas pelos futuros professores na sua prática pedagógica supervisionada.

\section{Formação inicial de professores de Informática na Universidade de Lisboa}

Na Universidade de Lisboa, a formação inicial de professores de Informática é da responsabilidade de duas das suas escolas sendo o Instituto de Educação responsável pelas componentes de formação educacional geral, didáticas específicas e iniciação à prática profissional e a Faculdade de Ciências responsável pela componente de formação na área de docência (Informática).

O plano de estudos prevê um conjunto de unidades curriculares (UCs) obrigatórias e quatro UCs optativas nas componentes de Formação Educacional Geral (FEG) e de Formação a Área de Docência (FAD). Na componente de FEG, os alunos podem optar por UCs como educação e media, educação para a cidadania, entre outras. Na componente de FAD podem selecionar UCs da especialidade de Informática como computação móvel, design de software, programação por objetos, etc.

As unidades curriculares opcionais na área da Informática visam proporcionar aos futuros professores oportunidades de complementar e atualizar a sua formação base, reforçando, deste modo, os seus conhecimentos no domínio da Informática. Na FEG organiza-se um leque de UCs que pretendem dotar o aluno de conhecimentos e competências na área da educação, nomeadamente em aspetos relacionados com a psicologia da educação, desenvolvimento e aprendizagem, avaliação, organização escolar e formação de adultos. Nas cinco UCs de didática específica (DE) procura-se trabalhar com os futuros professores questões pedagógicas, curriculares e didáticas do ensino de Informática. Nessas UCs abrange-se uma diversidade de áreas e temas do ensino de Informática, desde o ensino de hardware, programação, bases de dados ou robótica.

Por fim, na componente de formação de Iniciação à Prática Profissional (IPP) os futuros professores são envolvidos, ao longo de todo o seu percurso de formação inicial, em atividades de indução à prática docente em contexto real. Em cada uma das UCs de IPP, em especial a partir do $2^{\circ}$ semestre do $1^{\circ}$ ano, os futuros professores iniciam 
atividades letivas supervisionadas com alunos de turmas das escolas cooperantes neste processo através de protocolos estabelecidos com a ULisboa.

\section{Da Iniciação à Prática Profissional à Prática de Ensino Supervisionada}

A Iniciação à Prática Profissional (IPP) dos futuros professores de Informática ocorre ao longo de toda a sua formação inicial, ainda que com graus de intensidade diferentes. Como refere Formosinho (2001), é nos momentos de indução à prática profissional que os futuros professores aprendem a transformar os conhecimentos curriculares em conhecimentos profissionais suscetíveis de serem mobilizados na ação quotidiana de um professor. Considerando que existe um conjunto de saberes e de atitudes perante o conhecimento curricular numa determinada área, os hábitos de refletir e de questionar, inerentes ao desenvolvimento do pensamento crítico, constituem uma dimensão da prática necessariamente presente na formação dos futuros professores e, naturalmente, ao longo da sua vida profissional (Galvão \& Reis, 2005). Neste quadro, as UCs de IPP visam proporcionar oportunidades para que os futuros professores desenvolvam hábitos de análise, questionamento e reflexão sobre as (suas futuras) práticas docentes, permitindo-Ihes desenvolver atividades pedagógicas com alunos em contextos escolares diversificados.

Em IPP procura-se que os futuros professores observem e colaborem em situações de ensino e aprendizagem em contexto de sala de aula, desenvolvam experiência prática de planificação, ensino-aprendizagem e avaliação, participem no desenvolvimento de projetos e propostas pedagógicas e desenvolvam uma postura crítica e reflexiva sobre as atividades desenvolvidas.

Diversos autores têm devotado uma parte do seu trabalho de investigação à FIP apontando para a necessidade de ser proporcionada aos futuros professores a organização de momentos de indução na prática profissional de modo a promover a sua autonomia pedagógica e didática mas também o seu envolvimento na comunidade e no contexto escolar (Alarcão, 2005; Galvão \& Reis, 2005; Roldão, 2000; Sá-Chaves, 2000; Shulman, 1986). Esses momentos de prática profissional, abarcando uma diversidade das funções habituais do professor, ajudam a promover o desenvolvimento e identidade do futuro professor, constituindo-se como potenciadores da construção do seu saber profissional (Schön, 1987; Shulman, 1986).

É neste quadro que nas quatro UCs de IPP se procura proporcionar aos futuros professores experiências de formação diversificadas e com diferentes níveis de envolvimento em contexto escolar real.

No $2^{\circ}$ ano do curso de formação inicial de professores de Informática decorrem as últimas duas UCs de IPP, sendo no âmbito destas que os alunos desenvolvem os seus projetos de prática de ensino supervisionada. No âmbito desta prática, os futuros 
professores são supervisionados por dois professores orientadores, um da área da educação (IE ULisboa) e outro da área da Informática (Ciências ULisboa) e pelo professor cooperante da escola onde decorre a intervenção. Constitui-se assim uma equipa de trabalho com natureza multidisciplinar que trabalha com o futuro professor durante O $2^{\circ}$ ano nas UCs de IPP III e IPP IV.

Em IPP III são definidas as escolas cooperantes, as disciplinas e as temáticas nas quais os futuros professores irão desenvolver a sua prática de ensino supervisionada e são constituídas as equipas referidas acima. Após esta definição inicial, os futuros professores iniciam as suas atividades na escola, com duração de aproximadamente oito horas semanais. Numa primeira fase começam por abordar o contexto curricular e didático, analisando o currículo da disciplina que irão lecionar procurando identificar conceitos e processos a ensinar revendo aquilo que a literatura indica nesse campo. Esta análise visa identificar constrangimentos e dificuldades no ensino dos temas selecionados (e.g. ensino da programação) e estratégias e metodologias que podem ser utilizadas para colmatar essas dificuldades. Após o estudo curricular e didático, e com base nas observações das aulas do professor cooperante e no conhecimento sobre os alunos, o futuro professor inicia o desenvolvimento do seu plano pedagógico para a sua prática de ensino supervisionada, operacionalizado através do desenho de um Cenário de Aprendizagem (CdA). Procura-se, igualmente, nesta fase desenvolver todos os instrumentos de avaliação que irão ser mobilizados na sua prática de ensino, bem como identificar os recursos didáticos que será necessário desenvolver ou adaptar.

Durante a unidade curricular IPP IV decorre a implementação da prática de ensino supervisionada onde os futuros professores assumem a lecionação de um conjunto de aulas (no mínimo total de 480 minutos) correspondentes ao plano definido em IPP III.

Após terminarem a intervenção pedagógica, os estudantes redigem um relatório sobre a prática de ensino supervisionada que será presente para discussão perante um júri em provas públicas de mestrado.

\section{O papel estratégico dos cenários de aprendizagem}

Este artigo foca-se na relação entre o modelo pedagógico seguido na FIP, no que se refere à indução na prática pedagógica, e as opções pedagógicas realizadas pelos futuros professores na prática pedagógica supervisionada. Neste processo, o conceito de CdA assume um papel crucial.

Este conceito tem sido utilizado em diferentes áreas de atividade como estratégia para projetar o futuro, antecipando problemas e prevendo soluções para esses mesmos problemas. A utilização de cenários em situações de educação e formação apela ao desenvolvimento da capacidade de imaginar de forma fundamentada e sustentada situações educativas com objetivos claros e específicos (Pedro, Piedade \& Matos, 
2019). Identifica-se por isso uma relação natural entre essa capacidade e competências relacionadas com o pensamento crítico e criativo, a resolução de problemas, a comunicação e a colaboração, competências estas que têm vindo a ser recorrentemente referidas em vários referenciais internacionais (Pedro \& Matos, 2019).

A idealização de um CdA é algo que o professor faz regularmente, de forma mais ou menos sistematizada, sempre que planifica as suas atividades de ensino e procura antecipar ou desenhar diferentes experiências e problemas que pretende fazer acontecer na sua sala de aula no trabalho com os seus alunos. Deste modo, assume-se a ideia de CdA como estratégia para planificar atividades de ensino e aprendizagem em ambientes educativos enriquecidos com tecnologias digitais, em particular na formação inicial de professores.

Procurando definir o conceito, Matos (2014) refere que se trata de "uma situação hipotética de ensino-aprendizagem composta por um conjunto de elementos que descrevem (i) o contexto em que a aprendizagem tem lugar, (ii) o ambiente em que a mesma se desenrola e que é condicionado por fatores relacionados com a área de conhecimento, (iii) e os papéis desempenhados pelos diferentes agentes ou atores (e pelos seus objetivos), organizados numa narrativa" (p.3).

Outros autores referem-se aos CdA como ferramentas úteis para planear e descrever atividades de aprendizagem que promovam a aquisição de conhecimento na resolução de problemas reais e permitam aos alunos e professores saírem das suas formas pré-estabelecidas de pensamento e de atuação (Tetchung, Galartti \& Laube 2008; Wollenberg, Edmunds \& Bucke, 2000, Misfelf, 2015).

Matos (2014) considera um conjunto de elementos estruturantes presentes num CdA: (a) o desenho organizacional do ambiente - organização dos elementos contextuais do cenário, análise dos requisitos, seleção dos artefactos e dos materiais; (b) os papéis e os atores - definição das posturas e responsabilidades, dos modos de atuação, organização do coletivo e dos modos de interação e comunicação; (c) o enredo, as estratégias de trabalho, atuações e propostas de atividade - definição da arquitetura da atuação, da estrutura das propostas de atividade, da narrativa e do enredo; e (d) os mecanismos de reflexão, regulação e autorregulação - processos de reificação do aprendido, monitorização das aprendizagens e do processo de desenvolvimento próprio dos atores, avaliação crítica, avaliação dos produtos.

Um CdA assume um conjunto de características que podem funcionar como catalisadoras do desenvolvimento das competências para o séc. XXI atrás referidas (Matos, 2014): 


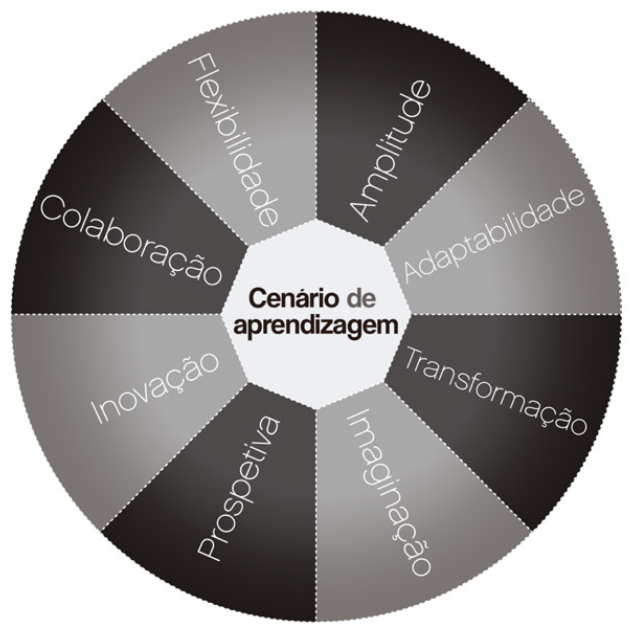

Figura 1. Características de um cenário de aprendizagem (Matos, 2014)

O processo de desenho e implementação de um CdA é condicionado por diversos fatores inerentes ao processo educativo, desde a área disciplinar e o domínio do conhecimento, passando pelos papéis dos diferentes intervenientes (e.g. alunos, professores, especialistas, convidados) até às sequências das atividades e tarefas de aprendizagem (Pedro et al, 2019). Sistematizando este processo, Matos (2014) definiu um conjunto de seis princípios orientadores que devem estar na base no processo de desenho de cenários de aprendizagem:

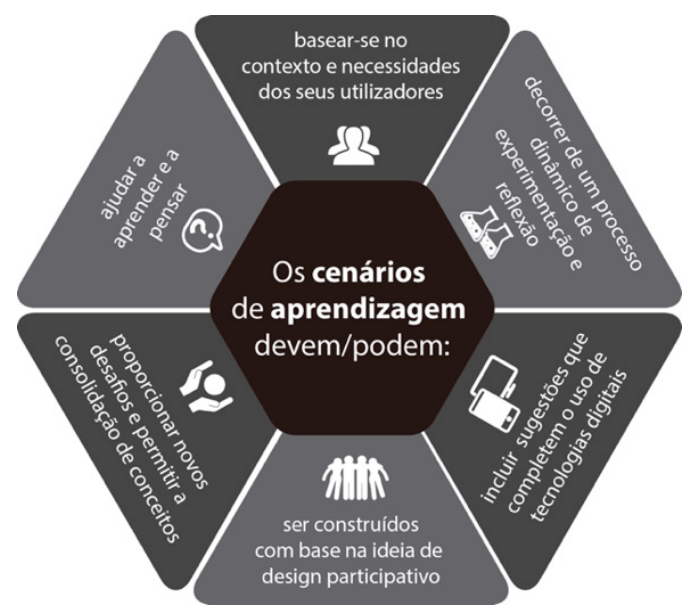

Figura 2. Princípios orientadores para o desenho de cenários de aprendizagem (Matos, 2014) 


\section{Metodologias ativas de aprendizagem na formação do professor de informática}

A prática de desenho de CdA, enriquecidos com tecnologias digitais, que recorrem a metodologias ativas de ensino e aprendizagem para a sua implementação em contextos escolares reais, tem vindo a ser consolidada ao longo dos últimos anos na formação inicial de professores de Informática na ULisboa. Ao longo do seu percurso formativo, os alunos tomam contato com várias metodologias ativas, os seus princípios, características e potencialidades pedagógicas bem como com atividades pedagógicas concretas de aprendizagem que recorrem a tais metodologias. Os relatos de prática pedagógica supervisionada desenvolvidos por estudantes em anos anteriores são organizadas em formato de videocases que documentam todo o processo de desenho e implementação de CdA e assim constituem recursos para a análise e reflexão por parte dos estudantes ${ }^{1}$.

A imersão dos futuros professores em práticas de ensino que apelem a formas pedagógicas ativas e inovadoras decorre nas próprias aulas e seminários que integram a parte curricular do curso de mestrado. É, assim, intencional o contacto dos futuros professores com as metodologias de ensino e de aprendizagem ativas no pressuposto de que a FIP desempenha um papel fulcral na estruturação de atitudes positivas dos professores em relação à valorização do papel dos alunos e ao reconhecimento da sua responsabilidade central na aprendizagem. Aquela imersão é realizada com suporte na utilização de tecnologias digitais e de metodologias inovadoras nas suas práticas letivas. Efetivamente, diferentes estudos têm salientado que professores em início de carreira que tiveram contacto com práticas pedagógicas inovadoras e com uso de tecnologias digitais na sua formação inicial, demonstram uma autoeficácia positiva na utilização das tecnologias em sala de aula (Brown \& Warschauer, 2006; Hammond, Fragkouli, Suandi, Crosson, Ingram, Johnston-Wilder, Johnston-Wilder, Kingston, Pope \& Wray, 2009; Paraskeva, Bouta \& Papagianna, 2008; Vongkulluksn \& Bowman, 2018). Assim, tendo por base elementos teóricos fundamentais sobre a aprendizagem, procura-se que os cenários de aprendizagem desenvolvidos pelos futuros professores de Informática sejam planeados recorrendo a metodologias ativas de ensino e de aprendizagem, tais como: (i) Aprendizagem Baseada em Projetos; (ii) Aprendizagem Baseada em Problemas; (iii) Pair Programming; ou (iv) Flipped Classroom.

Entende-se que a caracterização e descrição das práticas que os futuros professores de informática têm desenvolvido durante a sua formação possa trazer contributos essenciais para a perceção e análise da docência, contribuindo assim para um ensino inovador, eficaz e focado, quer no desenvolvimento de conhecimentos científicos e didáticos, quer como potenciador do desenvolvimento de competências para o século XXI. Deste modo, esta investigação tem como objetivos:

i. caracterizar o modelo pedagógico de iniciação à prática profissional baseado no desenho de cenários de aprendizagem na FIP; 
ii. analisar a relação entre esse modelo pedagógico e as práticas de ensino supervisionado dos futuros professores tal como são representadas nos respetivos relatórios.

Para atingir estes objetivos analisou-se: (i) a tipologia dos instrumentos utilizados na planificação das atividades pedagógicas dos futuros professores de informática, (ii) identificou-se as metodologias de ensino mobilizadas pelos futuros professores na prática de ensino supervisionada e (iii) a relação entre essas metodologias e a tipologia de tarefas e instrumentos de avaliação das aprendizagens utilizados.

\section{Metodologia}

Considerando os objetivos do estudo, procurou-se analisar as opções tomadas pelos futuros professores de informática nas práticas pedagógicas descritas nos relatórios de prática de ensino supervisionada do mestrado em ensino de informática (MEI) da ULisboa.

Para isso, incidiu-se a investigação numa análise bibliométrica e cienciométrica, procurando identificar as tendências encontradas e evidenciar simultaneamente áreas passíveis de desenvolvimento futuro (Raja \& Balasubramani, 2011). Adotou-se o conceito de cienciometria abordado por Tague-Sutcliffe (1992), incidindo o estudo na análise quantitativa da ciência, enumerando e analisando a estrutura da investigação, de forma a assinalar as tendências de uma determinada área.

Deste modo, assente numa abordagem quantitativa, esta investigação adotou um caracter descritivo e exploratório, já que se pretendeu conhecer e descrever um determinado fenómeno (Coutinho, 2008; Creswell, 2010). Como corpo de dados consideraram-se os relatórios de prática de ensino supervisionada do MEI realizados entre 2011 e 2018, analisados através duma métrica comum definida previamente, através da qual se procurou extrair e organizar dados que respondessem aos objetivos assumidos.

De forma a responder aos objetivos deste estudo realizou-se um levantamento de todos os relatórios de prática supervisionada no mestrado em Ensino de Informática entre 2011 e 2018 da ULisboa, ficando assim o corpus de dados com 56 publicações. Assim, analisaram-se estes relatórios identificados de acordo com os critérios e as categorias de análise definidos, tendo por base a informação facultada pelos autores em cada relatório.

\subsection{Critérios e categorias de análise}

Atendendo ao caráter descritivo da investigação, definiu-se uma estrutura de análise organizada em torno das seguintes categorias: (i) metodologias de ensino utilizadas, (ii) estratégia de planificação adotada, (iii) temáticas abordadas, (iv) natureza das tarefas e (v) métodos de avaliação. 
Metodologias e Estratégias de Ensino. A caracterização das estratégias de ensino utilizadas nos planos e cenários de aprendizagem constantes dos relatórios de mestrado analisados obedeceu à seguinte categorização: (i) Problem based learning; (ii) Project based learning; (iii) Flippded classroom; (iv) Inquiry based learning; (v) Gamification; (vi) Game based learning; (vii) Pair programming; e (viii) Modelos Instrutivos centrados no professor - Instrução direta. Estas categorias abrangem diferentes tipologias de organização genérica da atividade a realizar com os alunos.

Estratégia de planificação adotada. Para caraterizar a planificação que os futuros professores utilizaram, compreendendo assim como estes organizaram o planeamento das suas atividades a curto, médio ou longo prazo, a categorização das estratégias de planificação estruturou-se em: (i) Planificação a longo prazo (da totalidade da disciplina lecionada); (ii) Planificação a médio prazo (da unidade ou módulo); (iii) Planificação a curto prazo (planos de aula); (iv) utilização de Cenários de Aprendizagem; (v) Planificação de projetos; e (vi) outra.

Temáticas abordadas. Refere-se às temáticas científicas selecionadas para a prática de ensino supervisionada dos futuros professores: (i) Programação; (ii) Hardware/ Arquitetura Computadores; (iii) Bases de dados; (iv) Redes de Comunicação; (v) Word; (vi) Powerpoint; (vii) Excel; (viii) Multimédia; (ix) Segurança Digital; (x) Comunicação e Colaboração; (xi) Internet; (xii) Sistemas de Informação; (xiii) Outras.

Tarefas. Um elemento central na análise efetuada referiu-se às tarefas planificadas pelos futuros professores. Através da identificação da tipologia de atividades utilizadas poderá compreender-se o grau de inovação do plano ou cenário. Esta categoria estrutura-se em: (i) Fichas de Trabalho; (ii) Exercícios Guiados; (iii) Resolução de problemas; (iv) Pesquisa e Exploração; (v) Simulações; e (vi) Outras.

Avaliação. Para caracterização das opções pedagógicas feitas pelos futuros professores, é essencial compreender como estes organizaram a avaliação das aprendizagens dos seus alunos. Desse modo, procurou-se caracterizar como se organizava a avaliação, obedecendo à seguinte categorização: (i) Teste/atividade diagnóstico; (ii) Teste de Avaliação; (iii) Grelhas de observação; (iv) Grelhas avaliação projetos/produtos; (v) Fichas de Trabalho; (vi) Inquéritos; (vii) Grelhas de avaliação atitudes/comportamento; (viii) Grelhas de Avaliação por pares; (ix) Grelhas de autoavaliação; e, Diário de Bordo.

\section{Resultados}

Os 56 relatórios de prática de ensino supervisionada analisados, correspondentes aos trabalhos desenvolvidos pelos alunos do MEI da ULisboa entre 2011 e 2018, mostram que 22 trabalhos foram realizados por estudantes do género masculino e 34 por alunos do género feminino. Embora não constitua um elemento central a integrar 
na problemática analisada neste artigo, não pode deixar de se sublinhar a forte representação do género feminino (mais de 2/3) numa área de docência que, até ao final do século passado, constituía um domínio essencialmente masculino.

Uma prática que tem sido utilizada no MEl prende-se com o facto de os alunos beneficiarem, simultaneamente, de orientação pedagógica e de orientação científica em Informática no desenvolvimento da sua prática profissional supervisionada. Esta dupla orientação só foi implementada de forma completa em 2014/2015. Deste modo, dos 56 relatórios analisados, 23 (cerca de 41\%) corresponderam a alunos que tiveram dupla orientação (de Educação e de Informática).

Relativamente ao percurso formativo onde ocorreu a prática de ensino supervisionada, os dados obtidos mostram que a grande maioria das intervenções ocorreu em disciplinas de cursos no âmbito do ensino profissional.

Tabela 1 - Percurso formativo dos alunos com os quais decorreu a prática de ensino supervisionada

\begin{tabular}{lcc}
\hline \multicolumn{1}{c}{ Percurso Formativo } & n. & \% \\
\hline Ensino Regular & 11 & $19.64 \%$ \\
\hline Ensino Profissional & 42 & $75 \%$ \\
\hline Cursos de Educação e Formação (CEF) & 1 & $1.79 \%$ \\
\hline Cursos Vocacionais & 2 & $3.57 \%$ \\
\hline Educação e Formação de Adultos (EFA) & 0 & $0 \%$ \\
\hline
\end{tabular}

A maioria das intervenções ocorreu no correspondente ao ensino secundário, sendo que apenas $7 \%$ ocorreu no $3^{\circ}$ ciclo do ensino básico. Contudo, com a inclusão da disciplina de TIC no $2^{\circ}$ ciclo do Ensino Básico (de acordo com as alterações curriculares no ano letivo 2018/2019), poderão existir implicações futuras relativamente às disciplinas em que a prática de ensino supervisionada é realizada.

\section{Temáticas}

Os resultados mostram que a programação constitui o tema de trabalho na maioria das intervenções. Isto é indicador da importância do tema da programação na área de Informática, mas também revela uma escolha intencional por parte de muitos estudantes. A programação é uma temática que se presta especialmente para a realização de atividades de projeto e de resolução de problemas, uma vez que a programação pode ser entendida como uma ferramenta de trabalho de aplicação universal. Ao mesmo tempo, a escolha dos estudantes futuros professores é também reveladora de 
alguma apetência para a realização de projetos e de atividades de natureza aberta e investigativa com os alunos, refletindo as metodologias e abordagens pedagógicas utilizadas em muitas das UCs do mestrado, nomeadamente na dimensão de Didática de Informática. Esta relação é particularmente assinalável nos relatórios de prática de ensino supervisionada realizados pelos futuros professores que adotaram a ideia de cenário de aprendizagem.

\section{Metodologias de Aprendizagem}

É interessante notar que a quase totalidade dos futuros professores opte por abordagens pedagógicas que apelidam de Project Based Learning (PjBL) e de Problem Based Learning (PBL). É relevante notar que em diversas UCs do curso de mestrado, os docentes adotam metodologias de trabalho baseadas na resolução de problemas (PBL) em atividades ligadas essencialmente à área de didática da Informática. A fluência que os relatórios de prática de ensino supervisionada demonstram na discussão do PBL, e a própria adoção dessa metodologia, sugere uma clara relação entre as metodologias usadas pelos docentes no curso e aquelas que são selecionadas pelos futuros professores nas suas primeiras experiências em situação de sala de aula na escola. É de salientar também a adoção da estratégia pair programming por parte de alguns estudantes para a dinamização de atividade pedagógicas no ensino da programação. Embora com expressão muito reduzida, a abordagem através de flipped classroom é colocada como uma possibilidade, sobretudo no caso dos alunos dos anos finais do ensino secundário.

\section{Tarefas}

A seleção do tipo de tarefas a realizar durante a prática de ensino supervisionada orienta-se claramente para a pesquisa, exploração e resolução de problemas - aliás de forma coerente com a abordagem genérica de PBL ou PjBL adotada pelos futuros professores. Trata-se de tarefas desenhadas de acordo com a estrutura do projeto ou do problema definido como núcleo de desenvolvimento das atividades que os futuros professores preparam para a sua intervenção pedagógica. A pesquisa e exploração são suportadas pela utilização de tecnologias digitais - em particular com utilização de tecnologias móveis e acesso à internet - que são em geral integradas de forma natural nas atividades. Algumas exceções a esta naturalização na utilização das tecnologias digitais são referidas nos casos em que os alunos não têm uma prática habitual de uso na sala de aula. É importante referir que a prática de ensino supervisionada ocorre num número limitado de aulas (e.g. 10 a 15 aulas de 45') que constitui dessa forma uma 'janela' de experimentação por parte do futuro professor num contínuo de aulas que os alunos têm durante o ano com o professor cooperante titular da turma. Esta 
possível descontinuidade, quer nas atividades quer nos recursos tecnológicos que os alunos utilizam durante a prática de ensino supervisionada feita pelos futuros professores, pode explicar a forte adesão dos alunos ao tipo de tarefas que Ihes são propostas motivada pelo caráter de novidade gerador de curiosidade genuína.

\section{Planificação}

Os processos de planificação utilizados pelos futuros professores baseiam-se desde 2014 no desenho de cenários de aprendizagem. A estrutura de cenário - que inclui elementos também constantes dos processos tradicionais de planificação a longo prazo e aula-a-aula - permite criar uma visão da rede de conceitos e processos a ensinar que permite ao futuro professor desenhar com confiança (mas com responsabilidade) as atividades que melhor contribuirão para a aprendizagem dos alunos.

\section{Avaliação}

É surpreendente a percentagem reduzida de relatórios de prática de ensino supervisionada que apresentam o teste como instrumento fundamental de avaliação. Trata-se de um sinal da procura de alternativas à avaliação tradicional das aprendizagens provavelmente induzido pelo trabalho de análise e reflexão realizado em diversas UCs do curso de Mestrado. Simultaneamente, verifica-se que cerca de 73\% dos relatórios indica a utilização de instrumentos de observação e, aproximadamente o mesmo número, a utilização de instrumentos de avaliação de projetos e respetivos produtos. No caso da avaliação por pares, é indicada a sua utilização em cerca de 48\% dos relatórios, sendo 63\% aqueles que indicam processos e instrumentos de autoavaliação. Estes resultados consolidam a ideia de que os futuros professores procuram encontrar formas de organizar o processo de ensino e aprendizagem que coloque de forma coerente e alinhada as opções pedagógicas que realizam com as formas de avaliação das aprendizagens dos alunos.

\section{Conclusão}

Os resultados da análise dos relatórios de prática de ensino supervisionada apontam claramente para uma relação produtiva entre o modelo pedagógico seguido na formação inicial dos professores, no que se refere à iniciação à prática pedagógica, e as opções pedagógicas que os estudantes (futuros professores) realizam na prática pedagógica supervisionada. Pode concluir-se, tal como referido por Brown e Warschauer (2006), Ertmer, Ottenbreit-Leftwich, Sadik, Sendurur e Sendurur (2012), Matos, Pedro \& Pedro, 2017) ou Vongkulluksn et al. (2018), que a prática pedagógica dos docentes nos cursos preparatórios da FIP, tem uma influência importante nas opções 
pedagógicas que os futuros professores fazem ao delinear a suas primeiras experiências de ensino.

Esse facto suscita a questão do papel da dimensão da prática de ensino supervisionada na estrutura dos cursos de FIP, dimensão esta que é reconhecida como central na formação inicial dos professores (Blamire, Cassells \& Walsh, 2017). Mas coloca também a questão da duração do período de indução na prática profissional do futuro professor que, no caso analisado nesta investigação, é em média de 10 a 15 aulas de 45 minutos.

Na formação inicial de professores, a indução representa o suporte e orientação que os formadores necessariamente dão aos futuros professores. De um modo mais geral, a indução profissional inclui a orientação no local de trabalho, a dimensão da socialização profissional e, desejavelmente, o mentorado. A indução profissional de qualidade inclui uma formação continuada para além da formação inicial do professor, a formação de docentes para atuarem como mentores, tempo suficiente para que a atividade de mentorado produza efeitos duradoiros e processos de avaliação do desenvolvimento profissional que concorram para a autonomia profissional do professor.

De facto, uma verdadeira indução na prática profissional durante a formação inicial de professores sugeriria uma imersão com duração e continuidade bem maior que aquela que existe no curso de mestrado da Universidade de Lisboa. É por exemplo notória a segmentação que é realizada nas UCs de IPP separando (quer concetualmente quer na prática de observação de atividades escolares) as diversas dimensões da atividade do professor - desde a participação nos órgãos da escola, a análise dos programas no respetivo grupo disciplinar com vista ao desenho e planificação da atividade letiva, a análise e discussão em sede de conselho de turma dos casos de alunos com dificuldades de aprendizagem, etc. A indução profissional implica assim necessariamente uma imersão real na atividade profissional como sucede, por exemplo, na formação médica (Nóvoa, 2009). A segmentação dos períodos de contacto com a escola que coloca de fora a dimensão colaborativa das funções do professor - algo absolutamente central em qualquer atividade profissional. Esta é uma implicação que é importante sublinhar e que valerá a pena equacionar numa futura avaliação do modelo de formação inicial de professores.

\section{Notas}

1 http://ftelab.ie.ulisboa.pt/tel/gbook) 


\section{Referências Bibliográficas}

Alarcão, I. (2005). Professores reflexivos em uma escola reflexiva. São Paulo. Cortez Editora.

Brown, D., \& Warschauer, M. (2006). "From the university to the elementary classroom: Students' experiences in learning to integrate technology in instruction." Journal of Technology and Teacher Education, 14(3), 599-621.

Coutinho, C. \& Gomes, M. J. (2006). Critical review of research in educational technology in Portugal (2000-2005). Educational Multimedia and Hipermedia-Proceedings of EDMedia 06-World Conference on Educational Multimedia and Hipermedia, 2679-2686.

Creswell, J. W. (2010). Projeto de pesquisa. Métodos qualitativo, quantitativo e misto ( $3^{a}$ ed). Porto Alegre: Artmed.

Formosinho, J. (2001). A Formação prática dos Professores: da prática docente na instituição de formação à prática pedagógica nas escolas. In B. Campos (Org.), Formação profissional de professores no ensino superior. (pp. 46-64). Porto: Porto Editora.

Galvão, C. \& Reis, P. (2002). Um olhar sobre o conhecimento profissional dos professores: O estágio de Sofia. Revista de Educação, 11(2), 165-178.

Hammond, M., Fragkouli, E., Suandi, I., Crosson, S., Ingram, J., Johnston-Wilder. P., Wray, D. (2009). "What happens as student teachers who made very good use of ICT during pre-service training enter their first year of teaching?" Teacher Development, 13(2), 93-106.

Paraskeva, F., Bouta, H., \& Papagianna, A. (2008). "Individual characteristics and computer self-efficacy in secondary education teachers to integrate technology in educational practice". Computers \& Education, 50(3), 1084-1091.

Matos, J. F. (2014). Princípios orientadores para o desenho de cenários de aprendizagem. Lisboa: Instituto de Educação.

Matos, J. F., Pedro, N., \& Pedro, A. (2017). "Initial teachers' education practices within a technology enriched learning environment: Project FTELab". In M. J. Gomes, A. J. Osório, \& A. L. Valente, Livro de Atas da Conferência Internacional de TIC na Educação Challenges (pp.329-359). Braga: Instituto de Educação da Universidade do Minho. Available at http://www.nonio.uminho.pt/challenges/atas/.

Nóvoa, A. (2009). Para una formación de profesores construída dentro de la profesión. Revista de Educacion, 350, 203-218.

Pedro, A., Piedade, J., Matos, J.F. \& Pedro, N. (2019). Redesigning initial teacher's education practices with learning scenarios. The International Journal of Information and Learning Technology, 36 (3), 266-283 doi: https://doi.org/10.1108/IJILT-11-2018-0131.

Misfeldt, M. (2015). "Scenario based education as a framework for understanding students engagement and learning in a project management simulation game". The Electronic Journal of e-Learning, (13), 3, 181-191.

Raja, S. \& Balasubramani, R. (2011). Scientometric Study of the Research Publication on Malaria 20032007: A Global Perspective. International Research Journal of Library, Information and Archival Studies, 3 (1), 114-125. 
Sá-Chaves, I. (2000). Portfólios Reflexivos, Estratégias de Formação e de Supervisão. Cadernos Didácticos, Série Supervisão n¹. Aveiro: Unidade de Investigação Didáctica e Tecnologia na Formação de Formadores da Universidade de Aveiro.

Schön, D. (1987). Educating the reflective practitioner. Nova lorque: Jossey Bass.

Shulman, L. (1986). "Those who Understand: Knowledge Growth in Teaching”. Educational Researcher, 2 (15), 4-14.

Tague-Sutcliffe, J. (1992). An introduction to informetrics. Information Processing \& Management, 28 (1), 1-3.

Tetchueng, J., Garlatti, S., \& Laube, S. (2008). "A context-aware learning system based on generic scenarios and the theory in didactic anthropology of knowledge". International Journal of Computer \& Applications, 1 (5), 71-87.

Vongkulluksn, V. W., Xie, K., \& Bowman, M. A. (2018). The role of value on teachers' internalization of external barriers and externalization of personal beliefs for classroom technology integration. Computers \& Education, 118, 70-81. doi: https://doi.org/10.1016/j.compedu.2017.11.009.

Wollenberg, E., Edmunds, D., \& Bucke, L. (2000). "Using scenarios to make decisions about the future: anticipatory learning for the adaptive co-management of community forests". Landscape and Urban Planning, 1-2, ( 47), 65-77.

Ana Pedro

UIDEF, Instituto de Educação da Universidade de Lisboa

Email: aipedro@ie.ulisboa.pt ORCID: 0000-0001-7434-0321

João Piedade

UIDEF, Instituto de Educação da Universidade de Lisboa Email: jmpiedade@ie.ulisboa.pt ORCID: 0000-0002-4118-397X

João Filipe Matos

UIDEF, Instituto de Educação da Universidade de Lisboa

Email: jfmatos@ie.ulisboa.pt ORCID: 0000-0002-5546-5257

Data de submissão: Dezembro 2018

Data de avaliação: Março 2019

Data de publicação: Setembro 2019 